\title{
Agentenbasierte Modellierung und Simulation
}

\author{
Instrument prospektiver Technikfolgenabschätzung
}

\author{
Johannes Weyer, TU Dortmund, Fakultät Wirtschaftswissenschaften, Fachgebiet Techniksoziologie, Otto-Hahn-Str. 4, 44227 Dortmund \\ (johannes.weyer@tu-dortmund.de), Dorcid.org/0000-0002-0181-8723 \\ Michael Roos, Ruhr-Universität Bochum, Fakultät für Wirtschaftswissenschaften, Lehrstuhl Makroökonomik (michael.roos@rub.de), \\ (D) orcid.org/0000-0002-5465-9893
}

Der Beitrag führt in die Thematik ein und gibt einen Überblick über die theoretischen und methodischen Grundlagen der agentenbasierten Modellierung und Simulation (ABMS). Er stellt die Potenziale der neuen Methode dar und begründet, warum TA und ABMS von einer engeren Zusammenarbeit profitieren. Schließlich wirft er einen Blick auf die Beschränkungen der ABMS sowie auf aktuelle Herausforderungen.

Agent-Based Modeling and Simulation

A Tool for Anticipatory Technological Impact Assessment

This article introduces the topic of the special volume and gives an overview of the theoretical and methodological principles of agent-based modeling and simulation (ABMS). We present the potentials of the new method and argue why technological impact assessment and ABMS could benefit from a closer collaboration. Finally, we discuss the limitations of ABMS and current challenges.

KEYWORDS: ABMS, sociological theory, economic theory, methods

\section{Einleitung}

Die Technikfolgenabschätzung (TA) ist ein etabliertes und gut institutionalisiertes Instrument der Analyse technischer Innovationsprozesse und der durch diese induzierten gesellschaftlichen Folgen. Sie setzt dabei systemanalytische, diskursanalytische und beteiligungsorientierte Verfahren ein und versucht mit Hilfe prospektiver Verfahren, einen Blick in die Zukunft zu werfen (Grunwald 2010) bzw. zu verstehen, wie denkbare Zukünfte in der Gegenwart verhandelt werden (Lösch 2017). Szenariogeleitete Verfahren skizzieren unterschiedliche Entwicklungspfade und tragen auf diese Weise dazu bei, politische Entscheidungen

This is an article distributed under the terms of the Creative Commons Attribution License CCBY 4.0 (https://creativecommons.org/licenses/by/4.0/)

https://doi.org/10.14512/tatup.26.3.11 über die Entwicklung bzw. den Einsatz neuer Technologien zu unterstützen, beispielsweise im Bereich erneuerbarer Energien (Grabietz et al. 2016).

Für politische Entscheider kann es jedoch wichtig sein zu wissen, welche Faktoren den Ausschlag geben, dass der Entwicklungspfad eines komplexen soziotechnischen Systems sich in eine bestimmte Richtung bewegt, vor allem aber, welche Konsequenzen steuernde Interventionen haben. Hier kommt die agentenbasierte Modellierung und Simulation (ABMS) ins Spiel, die sich in den Wirtschafts- und Sozialwissenschaften seit einiger Zeit einer gewissen Beliebtheit erfreut, ermöglicht sie doch, komplexe Systeme im Computer zu modellieren und deren künftige Entwicklung zu simulieren (Gilbert 2008). Andere sozialwissenschaftliche Methoden wie Fallstudien, teilnehmende Beobachtung, Interviews oder fragebogengestützte Einstellungserhebungen liefern wertvolle Daten für die Entwicklung agentenbasierter Modelle, aber auch die Szenarien, mit denen die ABMS operiert. Man kann somit von einem komplementären Verhältnis unterschiedlicher Forschungsansätze sprechen, in das die ABMS sozioökonomischer Systeme die folgenden vier „Bausteine“ einbringt:

- Die ABMS ermöglicht eine dynamische Modellierung sozioökonomischer Systeme und damit eine Analyse von Prozessen des wirtschaftlichen und gesellschaftlichen Wandels.

- Grundlage der ABMS ist die Mikrofundierung sozioökonomischer Prozesse, d.h. die Agenten werden mit individuellen Eigenschaften, Präferenzen und Strategien ausstattet.

- Die Entstehung emergenter Strukturen auf der Makro-Ebene sozioökonomischer Systeme wird durch das Wechselspiel von Mikro-Ebene (Agenten) und Makro-Ebene (System) erklärt.

- Zudem ist die ABMS eine experimentelle Methode, die es erlaubt, Parameter gezielt zu variieren und die Auswirkungen der Variationen zu beobachten. Sie holt somit die Gesellschaft ins Labor und ermöglicht es, Experimente durchzuführen, die im Realmaßstab nicht praktikabel oder aus ethischen Gründen sogar verboten wären (Epstein 2007; Holtz et al. 2015). 
Die ABMS hat sich daher zu einem Instrument der wirtschaftsund sozialwissenschaftlichen Forschung entwickelt, das den Methodenkanon von qualitativen und quantitativen Verfahren ergänzt und erweitert (Van Dam et al. 2013) und so auch zu einer wertvollen Ressource der TA werden kann.

\section{Modellierung sozioökonomischer Systeme}

Ausgangspunkt der ABMS sind softwarebasierte Modelle, also abstrakte Repräsentationen realer soziotechnischer oder sozioökonomischer Systeme im Computer, z. B. des Verkehrs- oder des Wirtschaftssystems. In diesen Systemen bewegt sich eine große Zahl Agenten, die nach Maßgabe ihrer jeweiligen subjektiven Präferenzen autonom Entscheidungen treffen. Wichtig ist, dass diese Agenten miteinander interagieren. So können die Entscheidungen eines Agenten Verhaltensänderungen anderer Agenten nach sich ziehen. Gerade diese Interaktionen und die wechselseitigen Verhaltensanpassungen führen zu Ergebnissen, die ex ante schwer zu prognostizieren sind.

Beim Nachbau komplexer Systeme am Computer, insbesondere bei der Konstruktion der Agenten und der Entscheidungsund Interaktionsregeln greift die ABMS auf etablierte Theorien sozialen und/oder ökonomischen Handelns zurück. Dabei besteht eine Offenheit, welche Theorien und Modelle zum Einsatz kommen; sie müssen lediglich zwei Anforderungen erfüllen:

Zum einen müssen sie Annahmen über Regeln und Mechanismen enthalten (Hedström und Ylikoski 2010), z. B. über die Entscheidungsregeln der Akteure, über die Interaktions-Mechanismen sowie über die Verknüpfung von Mikro- und Makro-Ebene. Letzteres beinhaltet insbesondere die beiden Fragen, wie das System die Wahrnehmungen der Akteure beeinflusst (Makro-Mikro-Link) und wie umgekehrt aus den Handlungen der Akteure der Systemzustand emergiert (Mikro-MakroLink).

Zum anderen müssen sie formalisierbar sein, d.h. man muss die Regeln und Mechanismen in Computersprache übersetzen können.

Agentenbasierte Modelle sind ein sehr flexibles Analyseinstrument, das die Kombination von Annahmen aus verschiedenen Theorietraditionen erlaubt und gerade dadurch zu neuen Erkenntnissen führen kann. Durch die Formalisierung und Implementierung in einer Computersoftware lässt sich zudem überprüfen, ob eine Kombination verschiedener Theorien widerspruchsfrei möglich ist.

\section{Agentenbasierte Modelle}

Agentenbasierte Modelle bestehen in der Regel aus drei Komponenten: den Agenten, der Umwelt, in der sie sich bewegen, sowie den Regeln, denen die Interaktionen der Agenten mit anderen Agenten, aber auch mit der Umwelt folgen.

\section{Agenten}

Softwarebasierte Agenten sind durch interne Zustände wie Alter, Geschlecht etc. sowie durch Strategien (z. B. schnell vorankommen oder die Umwelt schützen) charakterisiert. Die Technik der objektorientierten Programmierung ermöglicht es, die Eigenschaften jedes einzelnen Agenten softwaretechnisch so zu kapseln, dass jeder Agent individuelle Eigenschaften hat; zudem können die Entscheidungsprozesse einer großen Zahl von Agenten nahezu simultan abgewickelt werden, so dass große Populationen heterogener Agenten am Bildschirm ,gezüchtet" und deren Interaktionsprozesse beobachtet werden können (Epstein und Axtell 1996, S. 14 f.; Resnick 1995, S. 42 ff.). Agenten können in vielen Dimensionen heterogen sein, wobei es vom jeweiligen Modellzweck abhängt, welche Dimensionen berücksichtigt werden. Sie können sich hinsichtlich ihrer Eigenschaften unterscheiden, beispielsweise in Bezug auf Wohnort, Geschlecht, Bildung, Wissen, Werte, Präferenzen, Einstellungen etc. In vielen Simulations-Modellen wird mit Agententypen gearbeitet, die typische Kombinationen dieser Eigenschaften beinhalten und damit z. B. die Schimankschen Akteurtypen (Schimank 2010) oder die Sinus-Milieus abbilden können (Jensen et al. 2016).

\section{Regeln}

Diese Eigenschaften beeinflussen, welche Entscheidungen die Agenten treffen, wie sie Informationen beschaffen und verarbeiten, welche Verhaltensstrategien bzw. Lernregeln sie verwenden und welchen Grad an Rationalität ihr Handeln hat. Schließlich kann auch abgebildet werden, dass Agenten unterschiedliche Netzwerke haben, innerhalb derer sie beispielsweise Informationen austauschen.

Jeder Agent orientiert sich bei seinen Entscheidungen an seinen individuellen Präferenzen und versucht, die aus seiner subjektiven Sicht geeignetste Lösung zu finden. (Die Entscheidungen können also ganz unterschiedlich ausfallen: Der eine fährt Rad, der andere Auto.) Die Agenten interagieren zudem mit ihrer Umwelt sowie anderen Agenten. Sie verändern durch ihre Aktionen die Randbedingungen des Handelns der anderen Agenten, indem sie z. B. zur Entstehung eines Verkehrsstaus beitragen.

\section{Topologie}

In einem agentenbasierten Modell muss auch eine Topologie bestimmt werden, d.h. auf welche Weise die Agenten miteinander verbunden sind und wer mit wem interagiert. Die Modelle können räumlich sein, wobei der Raum dann oft als zweidimensionales Raster in Form eines Schachbretts dargestellt wird. Denkbar ist aber auch eine geodätisch realistische Raumdarstellung mit an der Realität orientierten Verkehrs- und Kommunikationswegen. Alternativ kann die Topologie völlig auf räumliche Bezüge verzichten. Dann ist denkbar, dass zufällig bestimmt wird, welche Akteure miteinander in Kontakt stehen, oder nur solche Agenten interagieren, die in einem Netzwerk verbunden sind. Je nach Anwendung könnte das ein Verwandtschaftsnetzwerk sein, ein berufliches Netzwerk oder auch das Unternehmensnetzwerk, das deren Geschäftsbeziehungen abbildet. 


\section{Emergente Effekte, Interaktionen und Systemdynamik}

Durch die Interaktionen der Agenten entstehen emergente Effekte - überraschende und schwer vorhersehbare Systemzustände, die sich nicht aus den Eigenschaften der Systemelemente (also der Agenten) ableiten lassen, sondern das nicht-intendierte Ergebnis der intentionalen Handlungen einer Vielzahl autonom handelnder Agenten sind.

Ein anschauliches Beispiel für einen emergenten Effekt ist der Verkehrsstau. Niemand führt ihn absichtlich herbei, und dennoch entwickelt er sich nur deshalb, weil jeder an seiner Entstehung mitwirkt. Zudem hat er unerwartete Eigenschaften, die in den Regeln der Mikro-Ebene nicht zu finden sind: Die Verkehrsteilnehmer bewegen sich vorwärts, der Stau als Makro-Phänomen hingegen mit einer konstanten Geschwindigkeit in die entgegengesetzte Richtung - wobei die Teilnehmer wechseln (vgl. Resnick 1995, S. 41; Epstein und Axtell 1996, S. 33 35 sowie das Modell traffic basic in Wilensky 1997)

Das Ganze geschieht durch das Wechselspiel von Mikro- und Makro-Ebene dezentral-selbstorganisiert, ohne dass es eines sozialen Planers bedarf. Die Agenten sind in ihren Wahlhandlungen (choices) durch die strukturellen Bedingungen (constraints) geprägt, die ihrerseits das Resultat vorheriger Interaktionen eben dieser Agenten sind - und sich daher dynamisch wandeln. Im Unterschied zu den üblichen mathematischen Modellen in der Volkswirtschaftslehre findet auch keine Koordination des individuellen Verhaltens durch einen Fixpunkt des Systems wie ein Marktgleichgewicht statt. Die Agenten treffen ihre Entscheidungen autonom, ohne dass diese konsistent mit den Entscheidungen anderer Agenten sein müssen. Das bedeutet nicht, dass in agentenbasierten Modellen keine Gleichgewichtszustände auftreten können. Wenn es sie gibt, sind sie das endogene Ergebnis der dezentralen Entscheidungen; sie werden nicht durch die Annahme eines Gleichgewichts im System erzwungen. ${ }^{1}$

Die Dynamik des Systems - wie auch dessen Komplexität ergibt sich daher aus den Interaktionen der Agenten untereinander sowie mit ihrer Umwelt. Dabei folgen die Handlungen der Agenten oftmals einfachen Regeln (z. B. „,nimm die kürzeste Route“), erzeugen aber durch nichtlineare Interaktionen ein überraschend komplexes Resultat auf der Systemebene. Derartige nicht lineare Prozesse lassen sich mit keiner anderen Methode als der ABMS darstellen und untersuchen.

Darüber hinaus ist es möglich, steuernde Eingriffe in das System zu untersuchen, indem Interventions-Szenarien durchgespielt werden, deren Ziel z. B. die Verhinderung von Verkehrsstaus oder Blackouts in Stromnetz ist, die also ein globales Optimum zu erreichen versuchen, das mehr ist als die Summe der lokalen Optima sämtlicher Agenten, welches sich als Resultat dezentraler Koordination ergibt.

1 Analytische Modelle der VWL enthalten in der Regel Gleichgewichtsbedingungen, z. B. die Annahme, dass die angebotene und die nachgefragte Menge auf einem Markt gleich sind und der Markt daher geräumt ist.

\section{Realitätsbezug}

Wie bei jeder Modellierung ist keine hundertprozentige Abbildung eines Real-Systems möglich. Es muss stets eine Abstraktion erfolgen, welche die vielfältige Wirklichkeit auf Faktoren reduziert, die für die betreffende Fragestellung als relevant erachtet werden. Dies ermöglicht es, sich auf diese Teilaspekte zu konzentrieren und ausgewählte Themen gezielt zu bearbeiten. Im Unterschied zu rein mathematischen Modellen bestehen bei der Computermodellierung wesentlich mehr Freiheitsgrade, da die Einschränkung der mathematischen Lösbarkeit des Modells wegfällt. Dies ist ein Vorteil dieser Methode, da dadurch reichere und realitätsnähere Modelle möglich sind. Im Prinzip lassen sich beliebig komplexe Vorstellungen über Sachverhalte modellieren, so dass eine weitreichende Annäherung an die Realität denkbar ist. Die wesentlichen Beschränkungen der Modellierung liegen in der Rechenleistung des verwendeten Computers, der Verfügbarkeit von Daten zur Initialisierung und Parametrisierung des Modells und der Zeit, die zur Implementierung und Analyse des Modells zur Verfügung steht. Zugleich stellt diese Freiheit eine Herausforderung für den Modellierer dar, da nun aktiv entschieden werden muss, welcher Detailgrad gewählt werden soll, um den Zweck der Modellierung und Simulation mit angemessenem Aufwand zu erreichen.

\section{Software}

Um ein agentenbasiertes Modell zu implementieren, benötigen Entwickler eine Software. Grundsätzlich eignen sich dafür objektorientierte Programmiersprachen wie C++, Java oder Python. In einer Programmiersprache ist ein Objekt ein Element mit Funktionen, Methoden, Prozeduren und inneren Zuständen, das analog zu einem Agenten mit seinen Eigenschaften und seinem Verhalten ist. Die objektorientierten Programmiersprachen sind flexibel und vor allem schnell, was bei großen Modellen mit vielen Agenten ein Vorteil ist. Allerdings sind Programmiersprachen nicht einfach zu erlernen und können nicht bequem für die ABMS verwendet werden, da jeder Arbeitsschritt ,,von Hand" programmiert werden muss. Für Anwender, die keine ausgebildeten Programmierer sind, eignet sich daher spezielle ABMS-Software häufig besser, auch wenn solche Software oft langsamer und weniger mächtig ist als eine Programmiersprache. Es gibt mittlerweile eine Vielzahl von ABMS-Softwarepaketen, von denen AnyLogic, Lsd, Repast und Swarm recht bekannt sind.

In den Sozialwissenschaften ist das Programm NetLogo weit verbreitet, unter anderem weil es einen intuitiven Einstieg ermöglicht und sich daher auch gut für die Lehre eignet. Es erlaubt aber auch die Entwicklung avancierter Programme.

Vor gut zwanzig Jahren startete die ABMS-Community bei der Modellierung der Software-Agenten mit dem KISS-Prinzip (Keep It Simple, Stupid), wonach komplexe künstliche Gesellschaften aus einfachen Agentenregeln entstehen (Epstein und Axtell 1996). Der Ansatz, komplexe Aggregatzusammenhänge auf einfache Mechanismen und Verhaltensregeln zurückzufüh- 
ren, ist immer noch eine Strömung innerhalb der Complexity Science. Jedoch ist klar, dass sehr einfache Heuristiken menschliches Verhalten in Situationen, in denen bewusst Entscheidungen getroffen werden, nicht adäquat beschreiben. Die rasant gestiegene Rechnerleistung erlaubt es mittlerweile, auch Modelle zu implementieren, die auf sozial- oder wirtschaftswissenschaftlichen Handlungstheorien basieren und Entscheidungs-Algorithmen enthalten, die der Komplexität des Handelns und Entscheidens realer Entscheider nahekommen (Fink und Weyer 2011).

\section{Methode oder Theorie?}

ABMS-Konzepte erheben den Anspruch, nicht nur Methode zu sein, sondern darüber hinaus einen Beitrag zur sozial- und wirtschaftswissenschaftlichen Theoriebildung zu leisten, der zum einen darin besteht, Prozesse der Aggregation abzubilden, wie sie sich beim Übergang von der Mikro-Ebene der Akteur-Handlungen zur Makro-Ebene der sozioökonomischen Strukturen vollziehen (siehe dazu bereits Coleman 1995). Statistische Verfahren sowie formale Methoden der Netzwerkanalyse, die in der Ökonomie und der Soziologie weit verbreitet sind, können zwar statische Momentaufnahmen großer Kollektive liefern; aber die dynamischen Prozesse der Entstehung und Veränderung sozioökonomischer Systeme können sie nicht abbilden - und erst recht nicht erklären.

Der Beitrag der AMBS zur Theoriebildung besteht zum anderen darin, die basalen Mechanismen herauszufinden, die die Dynamik komplexer Sozialsysteme erklären können; aber diese Mechanismen werden nicht auf der Makro-Ebene der Strukturen von Wirtschaft und Gesellschaft, sondern auf der Mikro-Ebene des Handelns von Akteuren bzw. Agenten gesucht (siehe dazu bereits Hedström und Swedberg 1996). Epstein und Axtell behaupteten bereits 1996 in ihrem wegweisenden Buch „Growing Artificial Societies“, dass man soziale Systeme und deren Dynamik erst verstanden habe, wenn man sie nachbauen könne (,,can you grow it? “). Erst wenn es gelänge, künstliche Sozialsysteme zu schaffen, deren Verhalten demjenigen realer Systeme entspricht, dann habe man die Mechanismen verstanden, die die Systemdynamik produzieren. Dementsprechend denkt ihr Konzept bottom-up, also von den Interaktionen der Agenten ausgehend, anstatt top-down die Systemfunktionen in den Mittelpunkt zu rücken, wie es beispielsweise in der Systemtheorie Luhmannscher Prägung geschieht (Luhmann 1997). Allerdings besteht beim ,generativen Ansatz" von Epstein und Axtell ein Induktionsproblem: Selbst wenn bestimmte Annahmen auf der Mikro-Ebene ein in der Realität beobachtetes Verhalten auf der Makro-Ebene erzeugen können, ist damit nicht gezeigt, dass die Mikro-Annahmen notwendig für das zu erklärende Makro-Phänomen sind. Es wird damit eine mögliche Erklärung geliefert, die unter Umständen nur eine von vielen ist. Dennoch gilt: Selbst wenn die ABMS nicht beweisen kann, dass bestimmte Kausalketten die einzig möglichen Erklärungen realer Phänomene sind, besteht ihr Wert darin, die Dynamik komplexer soziotechnischer Sys- teme untersuchbar zu machen und zudem einen Beitrag zur Anwendung und damit auch zum Test der Plausibilität sozial- und wirtschaftswissenschaftlicher Theorien zu leisten.

Mit Hilfe der ABMS können Experimentatoren komplexe sozioökonomische Phänomene empirisch untersuchen und insbesondere nichtlineare Systemdynamiken beobachtbar machen. Einzelne Parameter lassen sich kontrolliert variieren und so Experimente mit unterschiedlichen Settings durchführen (z. B. ein Verkehrssystem mit bzw. ohne Förderung der Elektromobilität).

Auch die ABMS kann nicht in die Zukunft schauen, aber diese Methode ermöglicht es, Zukunftsszenarien am Computer durchzuspielen - ein entscheidender Vorteil gegenüber anderen Methoden. Etablierte Verfahren wie die fragebogengestützte Erhebung von Einstellungen können beispielsweise herausfinden, ob ein Befragter bereit wäre, vom Auto auf das Fahrrad oder den öffentlichen Personennahverkehr umzusteigen. Welche Folgen diese Einzelentscheidung hat und wie sich das ,,handelnde $\mathrm{Zu}$ sammenwirken“ (Schimank 2010) vieler Einzelner auf die Dynamik des gesamten Verkehrssystems auswirkt, kann mit Hilfe derartiger Verfahren jedoch nicht ermittelt werden. Die ABMS kann zudem aufzeigen, wie die Akteure - mit ihren sehr unterschiedlichen Einstellungen - auf externe Anreize reagieren (z. B. den Ausbau des Radwegenetzes) und wie dies sich wiederum auf die Systemdynamik auswirkt. Schließlich ermöglicht sie, Prozesse, die sich in Realsystemen über Jahre bzw. Jahrzehnte erstrecken würden, binnen kurzer Zeiträume zu simulieren.

\section{Nutzen der ABMS für die TA}

Simulationsexperimente werden damit $\mathrm{zu}$ einem wertvollen Instrument der Technikfolgenabschätzung und damit auch der Technologie- und Innovationspolitik, da sie die Option bieten, What-if-Szenarien auszuprobieren, also Entwicklungen sozioökonomischer Systeme unter bestimmten, variablen Prämissen zu analysieren. Die ABMS und die TA können so zu Partnern werden und sich gegenseitig ergänzen (Roos 2016). Zum einen können TA-Szenarien mit Hilfe der ABMS formalisiert und damit auf Vollständigkeit und Konsistenz überprüft werden, um implizite Annahmen, Argumentationslücken oder ggf. sogar interne Widersprüche aufzudecken.

Zum anderen können die durch die Simulation erzeugten What-if-Szenarien mit Experteneinschätzungen verglichen werden. Ein Mehrwert entsteht vor allem dann, wenn die Simulation zu überraschenden Ergebnissen führt, die von Experten nicht gesehen oder für unplausibel gehalten wurden. Die Simulation kann damit einen Anstoß geben, etabliertes Wissen oder konsensuale Einschätzungen in Frage zu stellen und den Rahmen dessen, was man zukünftig für möglich hält, zu erweitern.

Die ABMS trägt damit zu einem vertieften Verständnis komplexer Systeme bei. Sie hat zudem das Potenzial, Politiker und Stakeholder mit handlungsrelevantem Wissen zu versorgen, indem sie ihnen ein Instrument an die Hand gibt, um ein Gespür für die Wirkungen unterschiedlicher Interventionen $\mathrm{zu}$ entwi- 
ckeln. Sie kann Entscheidungsträger dafür sensibilisieren, dass auch für unwahrscheinlich gehaltene Technikfolgen unter bestimmten plausiblen Annahmen eintreten können und daher bedacht werden sollten. So wirkt sie einem Status-quo-Bias entgegen, wonach Entscheidungsträger geneigt sind zu erwarten, dass die Zukunft der Gegenwart ähnlich ist, was in nichtlinearen Systemen aber nicht der Fall sein muss.

\section{Limitationen}

Wie jede Forschungsmethode hat die ABMS Einschränkungen. Was im Einzelnen an der Methode kritisiert und als Schwäche gesehen wird, hängt vom wissenschaftstheoretischen Hintergrund des jeweiligen Kritikers ab.

\section{Artifizielle Modelle}

Forscher, die Theorien und Zukunftsszenarien diskursiv entwickeln und verbal darstellen, halten ABMS-Welten oftmals für artifiziell und übermäßig abstrakt. Aus dieser Perspektive gehen durch die formale Modellierung wichtige Details und Einsichten verloren (McDowall und Geels 2017). Dieser Einwand trifft jedoch jede Art formaler Modellierung und die ABMS sogar eher weniger als mathematisch-analytische Modelle. Im Vergleich zu mathematisch formulierten Gleichgewichtsmodellen in der Volkswirtschaftslehre ist die ABMS weniger abstrakt und realitätsnäher, was wiederum von manchen Ökonomen kritisiert wird (Midgley et al. 2007). Deren Argument lautet, dass die Realitätsnähe eines Modells nie ein Ziel an sich sein sollte. Ein gutes Modell müsse abstrakt und sparsam (parsimonious) sein, damit es möglichst allgemein gilt und sich auf die wirklich relevanten Faktoren beschränkt. Nach dieser Auffassung ist die ABMS oft zu komplex und unübersichtlich, so dass der Forscher die abgebildeten Zusammenhänge nicht in derselben Weise verstehen kann wie in einem mathematischen Modell (Lehtinen und Kuorikoski 2007).

Dass die ABMS mit gegensätzlichen Argumenten kritisiert wird, sehen wir als besondere Stärke der Methode: Vielleicht trifft gerade sie das richtige $\mathrm{Ma}$ an Detailreichtum und Abstraktheit und kann damit ein Bindeglied zwischen mathematischen und nichtmathematischen Forschungsansätzen sein.

\section{Validierung}

Ein Problem besteht in der Validierung der ABMS. Damit der Computer Ergebnisse berechnen kann, müssen die Modellparameter mit Zahlen versehen und die Anfangswerte der endogenen Variablen so festgelegt werden, dass bei den Simulationsläufen plausible Resultate entstehen. Dies ist nicht immer einfach, denn oft fehlen geeignete Datensätze, so dass die Daten erst aufwendig empirisch erhoben werden müssen. Zudem sind bestimmte Größen - z. B. Parameter in Lern- oder Nutzenfunktionen - nur schwer zu beobachten. Ein Modell kann dann als valide gelten, wenn es in der Lage ist, einen realen Ist-Zustand eines Systems am Computer zu reproduzieren, z. B. durch den
Abgleich mit historischen Daten zum Energiemix eines Landes (Van Dam et al. 2013, S. 126-130). Nur dann können die Aussagen, die es über ein reales System macht, als glaubwürdig gelten.

Aber trotz der Validierungsproblematik kann die ABMS für die TA sinnvoll und nützlich sein, wenn es um Zukunftsprognosen für komplexe Sozialsystemen geht, die immer mit gewissen Unsicherheiten verbunden sind. Gerade wenn Wissenschaftler nicht den Anspruch erheben, mithilfe einer ABMS quantifizierbare Wahrscheinlichkeitsaussagen über zu erwartende Technikfolgen zu machen, sondern lediglich Folgen zu antizipieren, die eintreten könnten, leistet die ABMS gute Dienste (Roos 2016). Unbekannte oder schwer zu bestimmende Parameter können zudem in Sensitivitätsanalysen systematisch variiert werden. Auf diese Weise ermittelt die ABMS, wie das System und seine Dynamik auf Änderungen der Parameter reagieren.

\section{Aktuelle Herausforderungen}

Seit der Gründung des Santa Fé Institute im Jahr 1984 wird in den Sozialwissenschaften mit der ABMS gearbeitet. Wie die Beiträge in diesem TATuP-Thema zeigen, hat die ABMS mittlerweile die Nische verlassen und ist zu einem viel diskutierten Bestandteil des Methodenkanons der Sozialwissenschaften geworden.

Eine aktuelle Herausforderung besteht darin, das Stadium der „Insellösungen“ zu überwinden und disziplinäre Standards (in Forschung und Lehre) zu schaffen - auch damit die Modelle vergleichbar und ihre Ergebnisse replizierbar werden. Die Kompatibilität vieler Modelle leidet oftmals daran, dass unterschiedliche Herangehensweisen bei der Modellierung gewählt werden, die ad hoc aus dem jeweiligen Projektkontext generiert werden und teils auch unzureichend begründete Annahmen über das modellierte Verhalten enthalten. Zudem fehlen bislang einführende Lehrbücher und Ausbildungsmaterialien. Und es mangelt an einem Konsens der Community über gute wissenschaftliche Praxis, z. B. hinsichtlich der Dokumentation der Modelle.

Eine große Herausforderung besteht schließlich darin, unterschiedliche Simulation Frameworks miteinander zu koppeln; insbesondere in interdisziplinären Projekten mit Ingenieurwissenschaftlern, die ihre eigenen Frameworks betreiben, ist es unabdingbar, Schnittstellen für die koordinierte Simulation zu schaffen, z. B. in Form der Co-Simulations-Plattform „mosaik“ (Steinbrink et al. 2017).

\section{Überblick über die Beiträge}

Das vorliegende TATuP-Thema stellt eine Reihe von ABMS-Studien vor, die aus dem Blickwinkel der Sozial-, Politik- und/oder Wirtschaftswissenschaften Beiträge zu Politik- und TA-relevanten Themen leisten. Behandelt werden unter anderem die nachhaltige Transformation des Verkehrssystems (Adelt und Hoffmann), die hier unter der Perspektive der Steuerung bzw. Umsteuerung komplexer soziotechnischer Systeme betrachtet werden. Darunter 
fällt auch die Frage, wie politische, wissenschaftliche und zivilgesellschaftliche Stakeholder potenziell konflikthafte Transformationsprozesse durch eine partizipative Governance gestalten können (Scheffran, Link, Shaaban, Süsser und Yang).

Zudem wird die Rolle thematisiert, die Märkte bei Innovationsprozessen spielen, etwa anhand plattformbasierter Märkte, in denen die Interaktionen der Marktteilnehmer durch eine dritte Instanz vermittelt werden (Heinrich und Gräbner). Mithilfe der ABMS kann zudem untersucht werden, wie sich die Marktteilnehmer durch strategisches Handeln wechselseitig beeinflussen und welche Auswirkungen dies auf den Markterfolg von Innovationen hat (Stummer und Günther). Dies gilt insbesondere im Fall von Öko-Innovationen, die sich auf etablierten Märkten nur dann durchzusetzen können, wenn sich die Konsumenten-Präferenzen wandeln (Lewalder). Daneben spielen auch Netzwerke eine wichtige Rolle, weil sie die Wissensdiffusion und Kooperation unterschiedlicher Akteure in regionalen Innovationssystemen fördern können (Müller, Kudic und Pyka).

Ziel dieses TATuP-Themas ist es, die TA-Community stärker mit dem Stand der ABMS in den Sozial- und Wirtschaftswissenschaften vertraut zu machen, Hinweise auf bislang zu wenig genutzte Synergiepotenziale zu geben, aber auch den Austausch innerhalb der ABMS-Community voranzutreiben. Die Breite der Themen und Ansätze der in diesem Heft vorgestellten Arbeiten zeigt, wie flexibel diese erfolgversprechende Forschungsmethode ist.

\section{Literatur}

Coleman, James S. (1995): Grundlagen der Sozialtheorie. Handlungen und Handlungssysteme. Band 1. München: Oldenbourg.

Epstein, Joshua M. (2007): Generative Social Science: Studies in Agent-Based Computational Modeling. Princeton: Princeton University Press.

Epstein, Joshua M.; Axtell, Robert (1996): Growing Artificial Societies. Social Science from the Bottom Up. Washington D. C.: Brookings Institution Press.

Fink, Robin D.; Weyer, Johannes (2011): Autonome Technik als Herausforderung der soziologischen Handlungstheorie. In: Zeitschrift für Soziologie 40 (2), S. 91-111. Online verfügbar unter http://www.zfs-online.org/index.php/zfs/ article/viewFile/3061/2598, zuletzt geprüft am 27.10.2017.

Gilbert, Nigel (2008): Agent-Based Models. Los Angeles: Sage.

Grabietz, Agnes; Trevisan, Bianka; Jakobs, Eva-Maria (2016): Lokale Akzeptanz von Energiemixen. In: TATuP - Zeitschrift für Technikfolgenabschätzung in Theorie und Praxis 25 (1), S. 61-66. Online verfügbar unter http://www.tatupjournal.de/tatup161_grua16a.php, zuletzt geprüft am 17.10.2017.

Grunwald, Armin (2010): Technikfolgenabschätzung. Eine Einführung. Berlin: Edition sigma.

Hedström, Peter; Swedberg, Richard (1996): Social Mechanisms. In: Acta Sociologica 39 (3), S. 281-308

Hedström, Peter; Ylikoski, Petri (2010): Causal Mechanisms in the Social

Sciences. In: Annual Review of Sociology 36, S. 49-67. D0I: 10.1146/annurev. soc.012809.102632.

Holtz, Georg; Alkemade, Floortje; de Haan, Fjalar et al. (2015): Prospects of Modelling Societal Transitions: Position Paper of an Emerging Community. In: Environmental Innovation and Societal Transitions 17, S. 41-58. DOI: 10.1016/j.eist.2015.05.006.
Jensen, Thorben; Holtz, Georg; Baedeker, Carolin; Chappin, Émile J. L. (2016): Energy-Efficiency Impacts of an Air-Quality Feedback Device in Residential Buildings: An Agent-Based Modeling Assessment. In: Energy and Buildings 116, S. 151-163. DOI: 10.1016/j.enbuild.2015.11.067.

Lehtinen, Aki; Jaakko Kuorikoski (2007): Computing the Perfect Model: Why Do Economists Shun Simulation? In: Philosophy of Science 74 (3), S. 304-329. DOI: $10.1086 / 522359$.

Lösch, Andreas (2017): Technikfolgenabschätzung soziotechnischer Zukünfte. Ein Vorschlag zur wissenspolitischen Verortung des Vision Assessments. In: TATuP - Zeitschrift für Technikfolgenabschätzung in Theorie und Praxis 26 (1-2), S. 60-65. D0I: 10.14512/tatup.26.1-2.60.

Luhmann, Niklas (1997): Die Gesellschaft der Gesellschaft. Frankfurt am Main: Suhrkamp.

McDowall, Will; Geels, Frank W. (2017): Ten Challenges for Computer Models in Transitions Research: Commentary on Holtz et al. In: Environmental Innovation and Societal Transitions 22, S. 41-49. DOI: 10.1016/j.eist.2016.07.001.

Midgley, David; Marks, Robert; Kunchamwar, Dinesh (2007): Building and Assurance of Agent-Based Models: An Example and Challenge to the Field. In: Journal of Business Research 60, S. 884-893.

Resnick, Michael (1995): Turtles, Termites, and Traffic Jams. Explorations in Massively Parallel Microworlds (Complex Adaptive Systems). Cambridge, MA: MIT Press.

Roos, Michael (2016): Modeling Radical Uncertainty and Anticipating Uncertain Change with Models. In: Forum for Social Economics, online veröffentlicht am 08. 09.2016. DOI: $10.1080 / 07360932.2016 .1229631$.

Schimank, Uwe (2010): Handeln und Strukturen. Einführung in eine akteurtheoretische Soziologie (4. Auflage). München: Juventa.

Steinbrink, Cornelius et al. (2017): Smart Grid Co-Simulation with MOSAIK and HLA: A Comparison Study. In: Computer Science - Research and Development, S. 1-9. DOI: 10.1007/s00450-017-0379-y.

Van Dam, Koen H.; Nikolic, Igor; Lukszo, Zofia (Hg.) (2013): Agent-Based Modelling of Socio-Technical Systems. Dordrecht: Springer.

Wilensky, Uri (1997): NetLogo: Traffic Basic Model. Evanston: Northwestern University. Online verfügbar unter http://ccl.northwestern.edu/netlogo/models/ TrafficBasic, zuletzt geprüft am 27.10.2017.

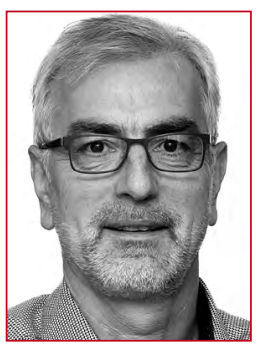

\section{PROF. DR. JOHANNES WEYER}

ist seit 2002 Professor für Techniksoziologie an der TU Dortmund mit Schwerpunkten in den Bereichen Steuerung komplexer Systeme, Mensch-Maschine-Interaktion, Netzwerkanalyse sowie ABMS.

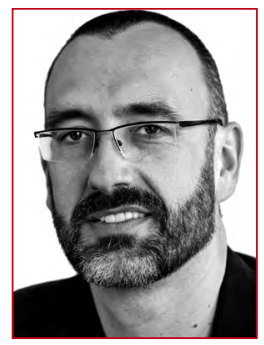

PROF. DR. MICHAEL ROOS

ist seit 2009 Inhaber des Lehrstuhls für Makroökonomik an der Ruhr-Universität Bochum und arbeitet vor allem in den Bereichen Komplexitätsökonomik, ABMS, Nachhaltigkeit und ökonomische Methodologie. 